\title{
A novel PHEX mutation associated with vitamin D-resistant rickets
}

\author{
Saori Sako' ${ }^{1}$ Yo Niida ${ }^{2}$, Kosuke Robert Shima', Yumie Takeshita', Kiyo-aki Ishii ${ }^{1}$ and Toshinari Takamura (iD)
}

\begin{abstract}
$X$-linked hypophosphatemic rickets $(X L H)$ is the most common form of hereditary rickets. Here, we present a case of $X \mathrm{LH}$ associated with a novel mutation in a phosphate-regulating gene with homologies to endopeptidases on the $X$ chromosome (PHEX). PCR-direct sequencing revealed a novel PHEX mutation in exon 22, NM_000444.6(PHEX):c.2202del [p.Asn736llefs*4], near the $3^{\prime}$-UTR region encoding the $\mathrm{COOH}$-terminal extracellular domain. In silico analysis indicated that a single mutation in N736 may have caused a significant change in higher-order protein structure and function.
\end{abstract}

$\mathrm{X}$-linked hypophosphatemic rickets $(\mathrm{XLH})$, the most common form of hereditary rickets, is associated with impaired renal tubular resorption that causes chronic hypophosphatemic rickets. A 2010 national epidemiological survey in Japan reported an estimated incidence rate of XLH of approximately 1 in 20,000 ${ }^{1}$. Hypophosphatemic rickets includes various diseases, such as vitamin D metabolite dysfunction, renal tubular abnormality, fibroblast growth factor 23 (FGF23)-related hypophosphatemia, and phosphorus deficiency. FGF23, which comprises 251 amino acids, decreases the expression of type $2 \mathrm{a}$ and type $2 \mathrm{c}$ sodium-phosphorus cotransporters, suppresses proximal renal tubular phosphorus reabsorption, and concurrently alters the expression of vitamin D metabolizing enzyme, resulting in a decrease in the concentration of $25-(\mathrm{OH})_{2}{ }^{2}$. FGF23-related hypophosphatemia is caused by genetic mutations in phosphateregulating gene with homologies to endopeptidases on the $\mathrm{X}$ chromosome $(P H E X)^{3}, F G F 23$, dentin matrix protein 1 (DMP1), ectonucleotide pyrophosphatase/phosphodiesterase (ENPP1), and family with sequence similarity 20 , member C (FAM20C). PHEX comprises 22 exons, encodes a type 2 transmembrane protein with a single-

Correspondence: Toshinari Takamura (ttakamura@med.kanazawa-u.ac.jp) 'Department of Endocrinology and Metabolism, Kanazawa University Graduate School of Medical Sciences, 13-1 Takara-machi, Kanazawa, Ishikawa 920-8640, Japan

2Division of Genomic Medicine, Department of Advanced Medicine, Medical Research Institute, Kanazawa Medical University, Ishikawa 920-0293, Japan pass structure and is primarily expressed in bones but not the kidneys ${ }^{4}$. Since a PHEX gene mutation was first identified in 1995, at least 364 mutations, including nonsense mutations, have been registered in the Human Gene Mutation Database (http://www.hgmd.cf.ac.uk/ac/ index.php). However, although PHEX mutations are thought to increase the production of FGF23, which promotes the phosphorus diuretic effect, resulting in hypophosphatemia, how PHEX gene mutations increase FGF23 and which domain is responsible for the function of PHEX remain unknown ${ }^{5}$. Therefore, understanding the relationship between mutation loci in PHEX and heterogeneous clinical features of XLH is necessary to better understand the function of PHEX in regulating FGF23 and phosphorus metabolism.

A 40-year-old Japanese woman was referred to our hospital for a detailed examination of her rickets. She had a short stature and bow legs but lacked a family history of congenital diseases and short stature. She had been born at full term via vaginal delivery. At 1 year of age, she experienced gait disturbance and was diagnosed with vitamin D-resistant rickets. Despite treatment with 1,25$(\mathrm{OH})_{2} \mathrm{D}_{3}$, her bone lesions worsened. At 25 years of age, her height was $131 \mathrm{~cm}$. X-ray examinations revealed genu varum and Looser's zones in the tibias. She underwent surgeries for bone correction and hip adductor muscle dissection. When the patient was 40 years of age, we performed laboratory tests, X-ray examinations of the 
Table 1 Laboratory data of the patient on admission

\begin{tabular}{lll}
\hline & Patient & Reference range \\
\hline$P(\mathrm{mg} / \mathrm{dL})$ & 1.8 & $2.5-4.5$ \\
$\mathrm{Ca}(\mathrm{mg} / \mathrm{dL})$ & 9 & $8.0-10.5$ \\
ALP $(\mathrm{IU} / \mathrm{L})$ & 255 & $115-359$ \\
$25-(\mathrm{OH}) \mathrm{D}_{3}(\mathrm{ng} / \mathrm{mL})$ & 12 & $7-41$ \\
$1.25-(\mathrm{OH})_{2} \mathrm{D}_{3}(\mathrm{pg} / \mathrm{mL})$ & 47.2 & $20-60$ \\
Intact PTH $(\mathrm{pg} / \mathrm{mL})$ & 62.4 & $10-65$ \\
BAP $(\mathrm{g} / \mathrm{L})$ & 23.6 & $2.9-22.6$ \\
FGF23 $(\mathrm{pg} / \mathrm{mL})$ & 42 & $<30$ \\
TmP/GFR $(\mathrm{mg} / \mathrm{mL})$ & 1.88 & $2.3-4.3$ \\
TRP $(\%)$ & 92 & $81-90$ \\
Estimated GFR $\left(\mathrm{ml} / \mathrm{min}\right.$ per $\left.1.73 \mathrm{~m}^{2}\right)$ & 161.89 & $\geqq 90$ \\
Urine Ca $(\mathrm{g} / \mathrm{day})$ & 0.116 & $0.1-0.3$ \\
\hline
\end{tabular}

lower limbs, computed tomography (CT) of the cervical spine, and measurements of bone mineral density (BMD).

Table 1 presents laboratory test data for the patient on admission. She had a phosphate (Pi) level of 1.8 (normal range, $2.5-4.5) \mathrm{mg} / \mathrm{dL}$, a calcium $(\mathrm{Ca}$ ) level of 9.0 (normal range, 8.0-10.5) $\mathrm{mg} / \mathrm{dL}$, an alkaline phosphatase (ALP) level of 255 (normal range, 115-359) IU/L, a bone-type ALP level of $82.4 \%$, an intact parathyroid hormone (PTH) level of 62.4 (normal range, 10.3-65.9) pg/mL, a 25hydroxyvitamin D3 [25- $(\mathrm{OH}) \mathrm{D}_{3}$ ] level of 12.0 (normal range, $7-41) \mathrm{ng} / \mathrm{mL}$, a $1,25-(\mathrm{OH})_{2} \mathrm{D}_{3}$ level of 47.2 (normal range, 20-60) $\mathrm{pg} / \mathrm{mL}$, an FGF23 level of 42 (normal range, $<30) \mathrm{pg} / \mathrm{mL}$, a tubular maximum phosphate reabsorption per glomerular filtration rate of 1.88 (normal range, 2.3-4.3) $\mathrm{mg} / \mathrm{dL}$, and an urine Ca level of 0.116 (normal range, $0.1-0.3) \mathrm{g} /$ day.

$\mathrm{X}$-ray examinations revealed genu varum and Looser's zones in the tibias, and cervical spine CT revealed calcification of the posterior longitudinal ligament of the spine with no evidence of tumors. Her BMD was $1.694 \mathrm{~g} / \mathrm{cm}^{2}$ at the lumbar spine (L2-L4).

Based on the findings of bone changes, hypophosphatemia, and elevated FGF23 levels, FGF23-related hypophosphatemia rickets was suspected; consequently, a genetic analysis was performed. Genomic DNA was extracted from whole blood samples using a genomic DNA extraction kit (Qiagen, Hilden, Germany). PCRdirect sequencing revealed a PHEX mutation, NM_000444.6(PHEX):c.2202del [p.Asn736llefs*4], in exon 22 (Fig. 1). This mutation was not found in various databases, including the Genome Aggregation Database (http://gnomad.broadinstitute.org), the Exome Aggregation Consortium database (http://exac.broadinstitute.org), and the Human Gene Mutation Database, suggesting that

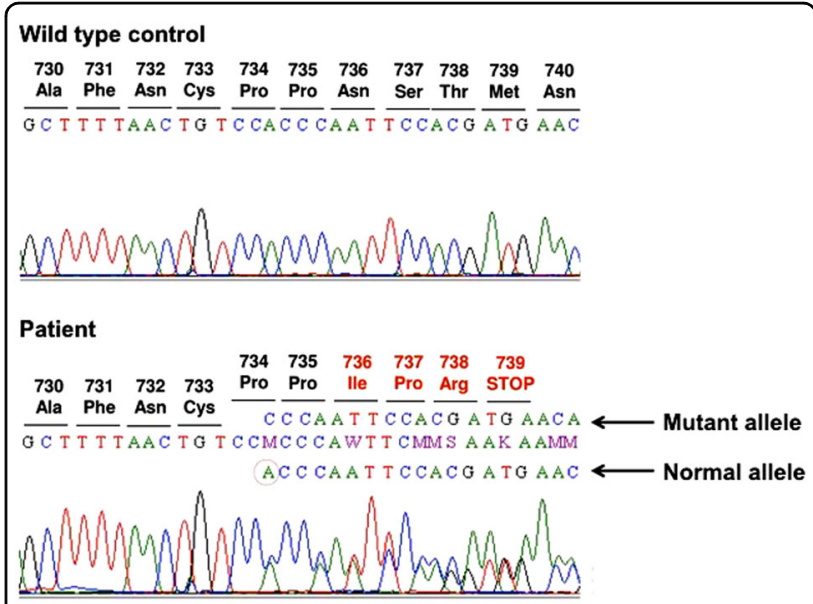

Fig. 1 Genomic DNA sequence of the patient's PHEX gene, indicating a novel mutation, NM_000444.6(PHEX):c.2202del [p.Asn736llefs*4], in exon 22

this mutation is novel rather than an SNP. Therefore, the patient's definitive diagnosis was XLH caused by a novel PHEX mutation. The mutation is believed to be "de novo", as assessed from a clinical perspective. She was treated with activated vitamin D metabolites and phosphate salts.

To our knowledge, we have described the first reported case of XLH caused by NM_000444.6(PHEX):c.2202del [p.Asn736Ilefs"4] in exon 22. This single base deletion induces a premature stop codon at position 739. Although this PHEX gene mutation is located near the $3^{\prime}$-UTR region encoding the $\mathrm{COOH}$-terminal extracellular domain, which contains no putative zinc binding sites or active sites, we believe that this mutation causes XLH based on the following reasons. First, the deleted region of the PHEX gene is highly conserved among wide ranges of mammalian species, with homology of $>88 \%$ for the $17 \mathrm{C}$ terminal amino acids in humans, mice, rats, rabbits, cats, dogs, horses, pigs, and bats. Furthermore, p.C733, p.C746, p.R747 ${ }^{6}$, and p.N736 (our case), which are mutated in patients with XLH, are completely conserved across species. These results suggest that this region is critical for the function of the protein. Second, we predicted the impact of this novel mutation on protein structure by conducting in silico analyses using software tools, including PROVEAN (http://provean.jcvi.org/index.php) and PANTHER (http://pantherdb.org/about.jsp); these in silico prediction engines produced scores of -4.43 and -3.05 , respectively, both of which suggested deleteriously damaged PHEX function. Third, in two other cases, XLH was reported to be associated with missense mutations located in the $\mathrm{COOH}$-terminal region of the PHEX gene $(\mathrm{C} 746 \mathrm{~W} \text { and } \mathrm{R} 747 \mathrm{X})^{6}$. Fourth, it is conceivable that even a single mutation causing a premature stop codon eliminates mRNA transcripts via the mechanism known as 
nonsense-mediated mRNA decay. Finally, our patient did not have any mutations in the genes encoding DMP1 and ENPP1, which are also involved in FGF23-related hypophosphatemia. In any event, because the protein encoded by the mutated PHEX is unknown, the causal phenotypic role of this mutation in rickets requires future study.

A limitation of the present report is lack of investigation of the mutant PHEX protein observed in the patient. As has been previously reported, it is necessary to investigate the trafficking, endopeptidase activity, and conformation of this protein ${ }^{7}$.

In summary, a single N736 mutation in the $\mathrm{COOH}$ terminal extracellular domain of PHEX may result in a significant change in higher-order protein structure and function and may cause XLH by increasing the production of FGF23. We believe that the present report contributes to understanding the relationship between mutation loci in the PHEX gene and heterogeneous clinical features of XLH.

\section{HGV Database}

The relevant data from this Data Report are hosted at the Human Genome Variation Database at https://doi.org/10.6084/m9.figshare.hgv.2522

\section{Conflict of interest}

The authors declare that they have no conflict of interest.

\section{Publisher's note}

Springer Nature remains neutral with regard to jurisdictional claims in published maps and institutional affiliations.

Received: 15 September 2018 Revised: 6 December 2018 Accepted: 9 December 2018.

Published online: 14 February 2019

\section{References}

1. Endo, I. et al. Nationwide survey of fibroblast growth factor 23 (FGF23) - related hypophosphatemic diseases in Japan: prevalence, biochemical data and treatment. Endocr. J. 62, 811-816 (2015)

2. Shimada, T. et al. FGF-23 is a potent regulator of vitamin D metabolism and phosphate homeostasis. J. Bone Miner. Res. 19, 429-435 (2004).

3. Francis, F. et al. A gene (PEX) with homologies to endopeptidases is mutated in patients with X-linked hypophosphatemic rickets. Nat. Genet. 11, 130-136 (1995).

4. Beck, L. et al. Pex / PEX tissue distribution and evidence for a deletion in the $3^{\prime}$ region of the Pex gene in X-linked hypophospha- temic mice. J. Clin. Invest. 99 1200-1209 (1997).

5. Yuan, B. et al. Aberrant Phex function in osteoblasts and osteocytes alone underlies murine X-linked hypophosphatemia. J. Clin. Invest. 118, 722-734 (2008).

6. Filisetti, D. et al. Non-random distribution of mutations in the PHEX gene, and under-detected missense mutations at non-conserved residues. Eur. J. Hum. Genet. 7, 615-619 (1999).

7. Sabbagh, Y., Boileau, G., Campos, M., Carmona, A. K. \& Tenenhouse, H. S. Structure and function of disease-causing missense mutations in the PHEX gene. J. Clin. Endocrinol. Metab. 88, 2213-2222 (2003). 